ECCOMAS

Proceedia
COMPDYN 2021

$8^{\text {th }}$ ECCOMAS Thematic Conference on Computational Methods in Structural Dynamics and Earthquake Engineering M. Papadrakakis, M. Fragiadakis (eds.) Streamed from Athens, Greece, 28 - 30 June 2021

\title{
GREEN BANK RADIO TELESCOPE: WIND INDUCED EFFECTS ON FEED-ARM
}

\author{
Gian Felice Giaccu ${ }^{1}$, Luca Gallisai ${ }^{2}$, Steven White ${ }^{3}$, Richard Prestage ${ }^{4}$, \\ Luca Caracoglia 5 \\ ${ }^{1}$ Department of Architecture, Design and Urban Planning, University of Sassari, \\ Alghero, Italy \\ e-mail: gf.giaccu@uniss.it \\ ${ }^{2}$ Department of Architecture, Design and Urban Planning, University of Cagliari, \\ Cagliari, Italy \\ ${ }^{3}$ Green Bank Observatory, National Radio Astronomy Observatory, \\ Green Bank, WV, USA \\ ${ }^{4}$ Department of Physics and Astronomy, West Virginia University, \\ Morgantown, WV, USA \\ ${ }^{5}$ Department of Civil and Environmental Engineering, Northeastern Univ., \\ Boston, MA, USA
}

\begin{abstract}
Radio Telescopes have been widely used in deep-space exploration and satellite communications. High pointing accuracy is required to be high in these applications, since the beams involved in these measurements are narrow. However, pointing precision can be influenced by wind load effects, which represent a disturbance source for the support structures of the telescope, especially in the high-frequency range. This paper analyzes the wind load effects on the feed arm of the Green Bank Radio Telescope (GBO) for two different wind directions as a function of the wind speed. A Finite Element (FE) Model of the GBO has been assembled to evaluate the dynamic response of the structure against turbulent wind disturbances. The FE model has been updated according to results derived from previously developed models. The paper focuses the attention on the wind induced displacements of the feed arm, which is, for the examined structure, the main source of wind disturbances. The study shows that wind disturbances can deeply affect the pointing accuracy of $G B O$ even at low wind speeds. Namely, for reference mean wind speed of $4 \mathrm{~m} / \mathrm{s}$ the total along-wind lateral displacements of the feed arm can reach the maximum threshold associated with an acceptable Pointing Error (PE). Furthermore, dis-placements are larger for winds acting in the cross-elevation direction and can deeply affect pointing accuracy of the antenna measurements.
\end{abstract}

Keywords: Radio Telescopes, Large reflector antennas, Wind Turbulence, Wind induced Disturbances. 


\section{INTRODUCTION}

The Green Bank Radio Telescope is a large reflector antenna located in West Virginia, United States; it is a fully steerable, $100 \mathrm{~m}$ diameter paraboloidal radio telescope capable of operating with high efficiency in a wide frequency range: from 0.1 to $116 \mathrm{GHz}$ (wavelengths $3.0 \mathrm{~m}-2.6 \mathrm{~mm}$ ). The telescope is located near the heart of the United States National Radio Quiet Zone, a unique area in North America, close to the city of Green Bank, West Virginia, where authorities restrict all radio broadcasts to avoid emissions interfering with the GBT itself. The position of the telescope inside the zone of radio silence allows the detection of radio frequency signals, avoiding the interference of other weak signals emitted by other sources or human activities. The observatory is bordered by the National Forest and the Allegheny mountains, which shield it from further radio interference. As for the large antennas, which usually operate in open space without any protection from atmospheric loads, variable wind load effects can influence the accuracy of the pointing; in fact, the wind interference becomes one of the causes of PE that can induce structural resonance at low natural frequencies (close to $1 \mathrm{~Hz}$ ).

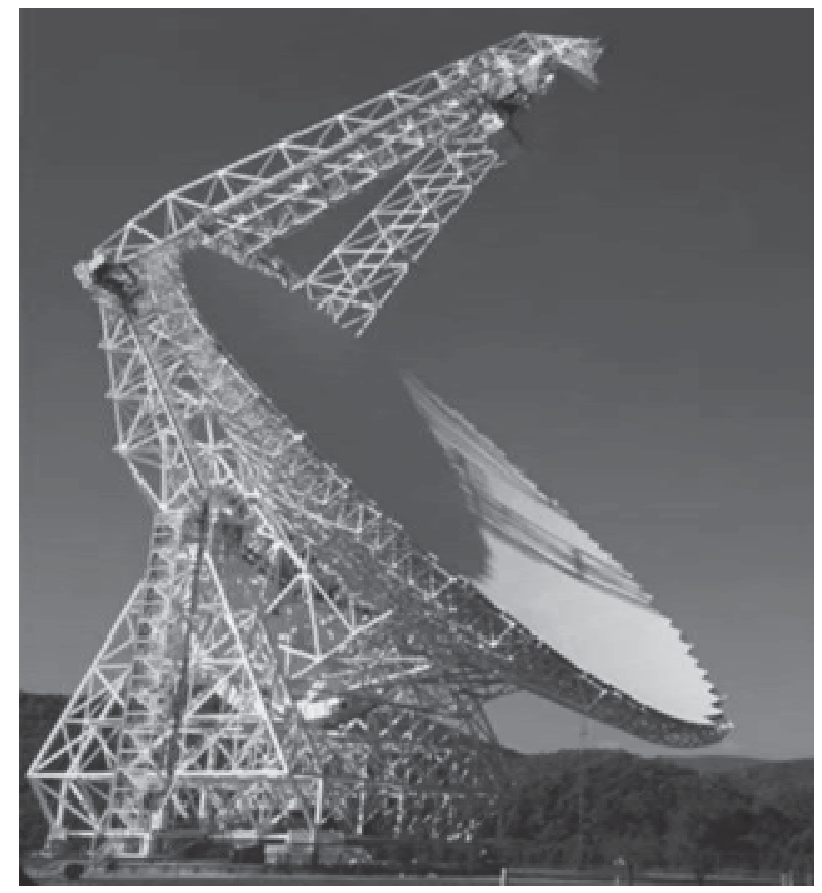

Figure 1. Picture of Green Bank Radio Telescope (reproduced from www.skyandtelescope.com)

Pointing accuracy of large reflector antennas is influenced by few factors: deformation of the main reflector, lateral movement of the Feed-Arm, lateral displacement of the subreflector, rotation of the sub-reflector around the point of focus. Several types of orientation errors may be observed in large telescopes, requiring complex adjustment mechanisms to minimize the undesirable effects. These conditions can be, either integrally or in part, a consequence of the wind loads.

Pointing errors due to wind excitation have been often observed but rarely examined in the literature since the problem is particular to each structure (and telescope). For example, the first documented case of significant wind load effects on PE was documented by Ulich [1]. The current state-of-the-practice and analysis approach considers the combination of wind tunnel tests and computational fluid dynamics to simulate the wind loads [2-4]. Since each 
structure is unique and effects are on occasion not fully predicted by simulations (i.e. during design), measurements of the flow field and wind loads around full-scale telescopes have also been considered [5]. In the case of flexible telescopes the issue of large deformation in the antenna may also affect the structural response [6]. Despite all these efforts, several problems are still unresolved and require careful consideration.

In this work the focus has been given to the lateral displacement of the sub-reflector, located at the top of the feed-arm, since it can be considered the largest source of wind induced PE in GBO. This work is part of ongoing research activity, which analyzes the wind-induced PE on large radio telescopes.

\section{DESCRIPTION OF FINITE ELEMENT MODEL AND WIND LOAD SIMULA- TION}

\subsection{Finite Element (FE) model of GBO}

For the purpose of studying wind effects on GBO, an FE model has been built, using in Strand 7 software package. The model consists of a total of 5702 nodes, 6133 beam elements, 2477 plate elements, 968 brick elements (Figure 2). Despite the geometric complexity of the model, the computational aspects do not require special efforts. Various models aimed to evaluate several load conditions, such as temperature gradient or gravitational effects, were previously built for similar structures [7].

The proposed analysis methodology is based on the application of wind loads on the structure through the FE model, after suitable discretization of the feed-arm in different sectors, each having different pressure distributions [8]. The discretization is schematically illustrated in Figures 2b, 2c. This approach aims to assess the wind load effects on the structure in terms of local displacements of the sub reflector, located at the top of the feed arm and the pointing error (PE), which represents the angle of rotation of the antenna compared to the nondeformed configuration [9-11].
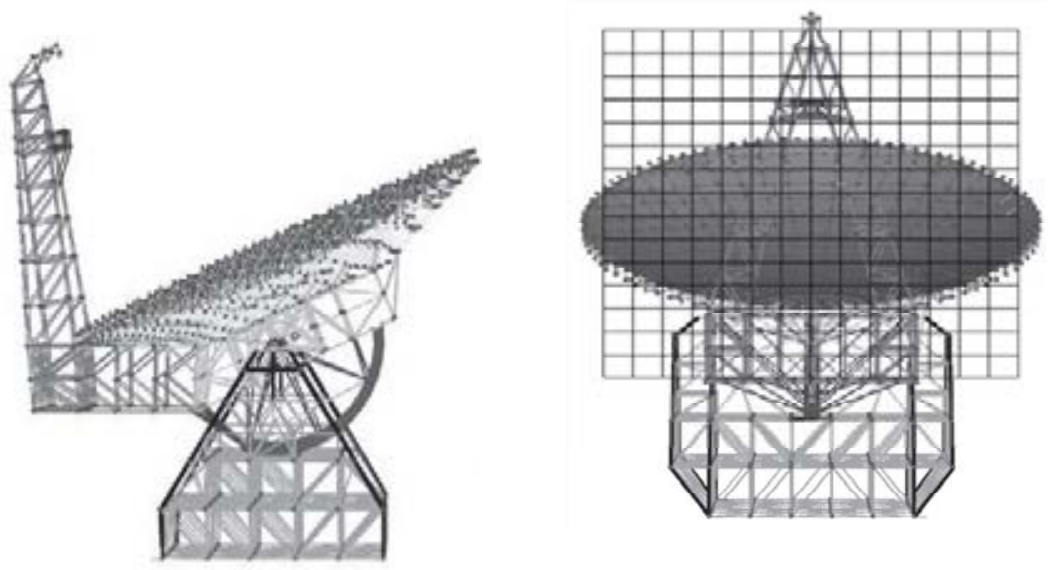

(a)

(b)

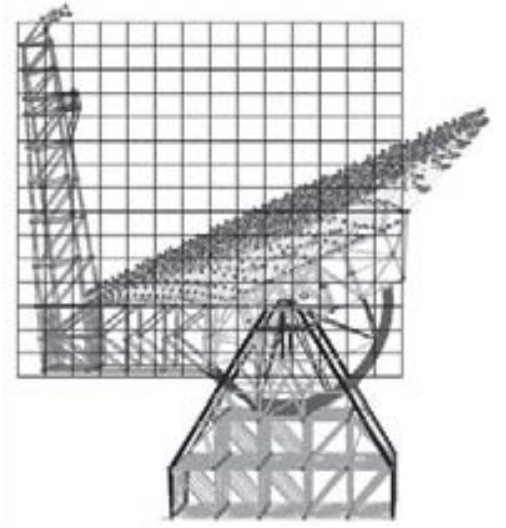

(c)

Figure 2. (a) Finite element model of the Green Bank Radio Telescope, Schematic representation of the grid utilized for simulating wind forces on feed-arm, (b) elevation direction, (c) cross-elevation direction.

\subsection{Application of the wind forces on the model of GBO}

Wind load simulation has been studied on the GBO through spatial discretization of the wind field using a $15 \times 15(6 \mathrm{~m} \times 5 \mathrm{~m})$ idealized grid in front of the telescope. At each node of the grid partially-correlated wind turbulence time histories of the along-wind field (primary) 
load component are synthetically generated by standard wave superposition method [12]. The turbulent wind load, generated by assuming validity of the quasi-steady aerodynamic theory, which can be used to study a variety of structures (e.g. [13, 14]), has been imposed to simulate pressures and equivalent forces (based on tributary areas). The pressure coefficients of the telescope surface have been extracted and adapted from the results by Liu Yan [8]. The synthetically generated time histories of the loads have a duration equal to $60 \mathrm{~s}$, with a time step $\Delta \mathrm{t}=0.01 \mathrm{~s}$. The loads have been evaluated for both "elevation" and "cross-elevation" mean wind directions, as shown in Figure 3.

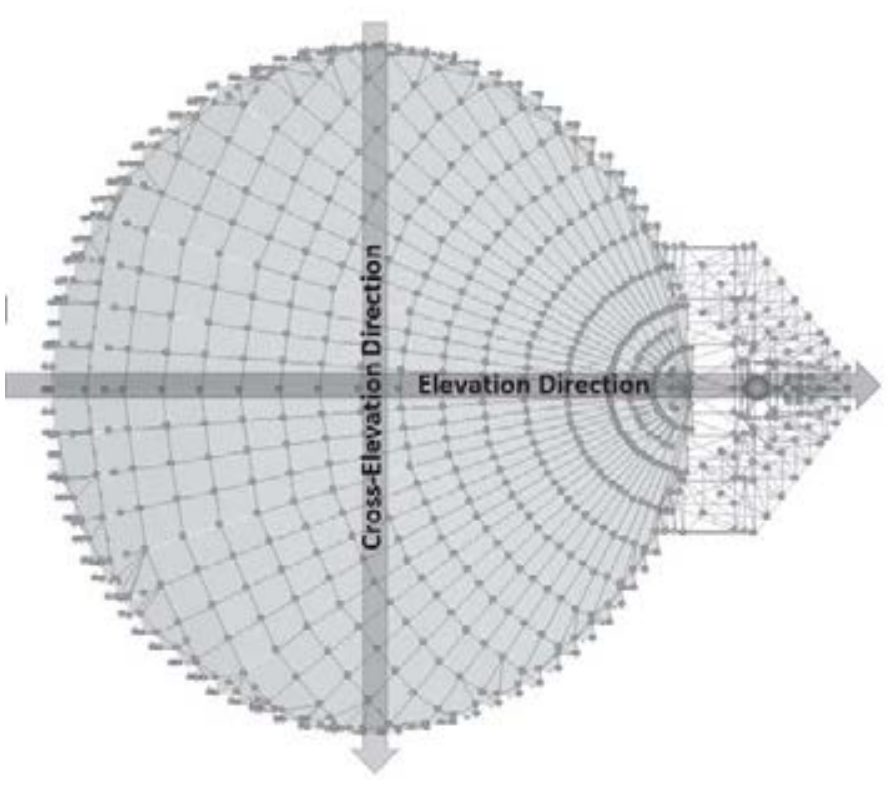

Figure 3. Schematic representation of main, incident wind directions.

\subsection{Linear dynamic integration approach}

A linear, (step-by-step) dynamic integration approach has been applied for examining the wind load effects, described in the previous sections and acting on the feed arm, for the two examined mean wind directions (Figure 3). The Results of the dynamic integration approach of wind load simulation are illustrated in Figure 4 for a reference mean wind speed of $4 \mathrm{~m} / \mathrm{s}$ at a reference elevation from ground $\mathrm{z}_{\mathrm{ref}}=10 \mathrm{~m}$.
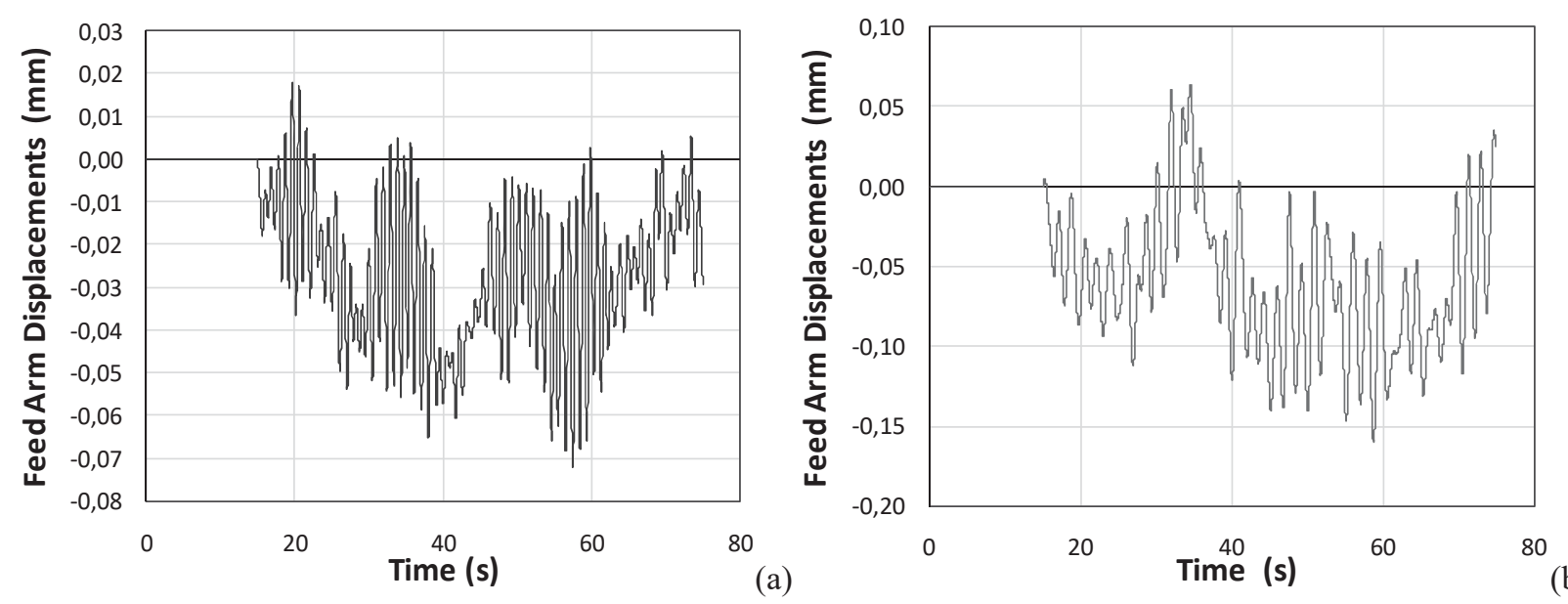
Figure 4. Time history of turbulent along-wind dynamic displacements of the top node of feed-arm, for wind acting in (a) elevation direction and (b) cross- elevation direction, at a reference ground speed of $4 \mathrm{~m} / \mathrm{s}$.

Figure 4a presents typical along-wind displacements of the feed-arm top node for mean wind acting in the elevation direction; Figure $4 \mathrm{~b}$ shows an example of along-wind displacements for mean wind acting in the cross-elevation direction.

\section{CONCLUSIONS}

This study discusses the turbulent wind effects on the GBO for two different wind directions: elevation and cross-elevation direction. Along-wind induced displacements of the feedarm were examined for different ground wind speeds. Results shows that, even for low ground wind speed such as $4 \mathrm{~m} / \mathrm{s}$, displacements of the-feed arm can approach the maximum threshold for pointing PE. In particular, displacements are greater for the wind acting in crosselevation direction. Future studies will examine the validity of the quasi-steady aerodynamic theory for the estimation of wind loads, the relevance of transverse loads due to wake excitation along with potential flow-structure interaction.

\section{ACKNOWLEDGEMENTS}

The first author would like to acknowledge the financial support of Regione Autonoma della Sardegna (L.R. n. 3/2008 "Rientro Cervelli" and L.R. n. 7/2007 "Promozione della Ricerca Scientifica e dell'Innovazione Tecnologica in Sardegna").

\section{REFERENCES}

[1] B.L. Ulich, Pointing errors of large telescopes due to wind, Very Large Telescopes, their Instrumentation and Programs., European Southern Observatory (IAU Colloquium n 79), Garching, Garching, W Ger, 1984, pp. 177-181.

[2] D.G. MacMynowski, K. Vogiatzis, G.Z. Angeli, J. Fitzsimmons, J.E. Nelson, Wind loads on ground-based telescopes, Applied Optics 45(30) (2006) 7912-7923.

[3] M. Mamou, K.R. Cooper, A. Benmeddour, M. Khalid, J. Fitzsimmons, R. Sengupta, Correlation of CFD predictions and wind tunnel measurements of mean and unsteady wind loads on a large optical telescope, Journal of Wind Engineering and Industrial Aerodynamics 96(6-7) (2008) 793-806.

[4] M. Mamou, A. Tahi, A. Benmeddour, K.R. Cooper, I. Abdallah, M. Khalid, J. Fitzsimmons, Computational fluid dynamics simulations and wind tunnel measurements of unsteady wind loads on a scaled model of a very large optical telescope: A comparative study, Journal of Wind Engineering and Industrial Aerodynamics 96(2) (2008) 257-288.

[5] G.Z. Angeli, M.K. Cho, M. Sheehan, L.M. Stepp, Characterization of wind loading of telescopes, Workshop On Integrated Modeling of Telescopes, February 5, 2002 - February 7, 2002, SPIE, Lund, Sweden, 2002, pp. 72-83.

[6] Z. Cheng, C. Ma, Distributed real-time networked control architecture for suppressing wind load deformation of Fourier telescope, 2014 6th International Conference on Intelligent Human-Machine Systems and Cybernetics, IHMSC 2014, August 26, 2014 - August 27, 2014, Institute of Electrical and Electronics Engineers Inc., Hangzhou, China, 2014, pp. 364-367.

[7] F. Buffa, A. Causin, A. Cazzani, S. Poppi, G. Sanna, M. Solci, F. Stochino, E. Turco, The Sardinia Radio Telescope: A comparison between close-range photogrammetry and finite element models, Mathematics and Mechanics of Solids (2015) 1-22.

[8] Q.H. Liu Yan, Fan Feng, Wind load characteristics of large radio telescope, Infrared and Laser Engineering 44(1) (2015) 148-156. 
[9] J. Zhang, J. Huang, L. Qiu, R. Song, Analysis of Reflector Vibration-Induced Pointing Errors for Large Antennas Subject to Wind Disturbance: Evaluating the pointing error caused by reflector deformation, IEEE Antennas \& Propagation Magazine 57(6) (2015) 46-61.

[10] F. Wang, R. Prestage, R. McCullough, G.-S. M., Green Bank Telescope Sub-reflector Stabilization (Wind), Metrology and Control of Large Telescopes, Green Bank, West Virginia, USA, 2016.

[11] L. Gallisai, Green Bank Telescope: valutazione del pointing error determinato dall'azione turbolenta del vento, University of Cagliari, Italy, 2018.

[12] M. Di Paola, Digital simulation of wind field velocity, Journal of Wind Engineering and Industrial Aerodynamics 74-76 (1998) 91-109.

[13] C. Borri, C. Costa, Quasi-steady analysis of a two-dimensional bridge deck element, Computers \& Structures 82(13-14) (2004) 993-1006.

[14] P.J. Richards, R.P. Hoxey, Quasi-steady theory and point pressures on a cubic building, Journal of Wind Engineering and Industrial Aerodynamics 92(14-15) (2004) 1191-1218. 